\title{
Concordancia entre padres e hijos en la calidad de vida relacionada con la salud en niños con trastorno por déficit de atención con hiperactividad: estudio longitudinal
}

\author{
L. Rajmil ${ }^{a, b, c, *}$, M.D. Estrada ${ }^{a, c}$, M. Herdman ${ }^{b, c}$, V. Serra-Sutton ${ }^{a}$, C. Tebé ${ }^{a, c}$, J. Izaguirre ${ }^{d}$, \\ J.A. Alda ${ }^{d}$, J. Alonso ${ }^{b}$, A.W. Riley ${ }^{e}$, C.B. Forrest ${ }^{f}$ y B. Starfield ${ }^{e}$
}

\author{
${ }^{a}$ Agència d'Avaluació de Tecnologia i Recerca Mèdiques, Institut Municipal d'Investigació Mèdica (IMIM-IMAS), \\ Barcelona, España \\ bInstitut Municipal d'Investigació Mèdica (IMIM-IMAS), Barcelona, España

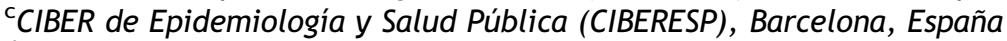 \\ ${ }^{\mathrm{d} S e r v e i ~ d e ~ P s i q u i a t r i a ~ i ~ P s i c o l o g i a, ~ H o s p i t a l ~ d e ~ S a n t ~ J o a n ~ d e ~ D e ́ u, ~ B a r c e l o n a, ~ E s p a n ̃ a ~}$ \\ e Johns Hopkins School of Public Health, Baltimore, Estados Unidos de América \\ ${ }^{f}$ Children's Hospital of Philadelphia, Philadelphia, Estados Unidos de América
}

Recibido el 14 de noviembre de 2008; aceptado el 10 de diciembre de 2008

Disponible en Internet el 7 de mayo de 2009

\section{PALABRAS CLAVE \\ Calidad de vida relacionada con la salud; \\ Concordancia entre padres e hijos; Trastorno por déficit de atención con hiperactividad}

\begin{abstract}
Resumen
Objetivos: Evaluar la concordancia entre padres e hijos sobre los cambios producidos en la calidad de vida relacionada con la salud (CVRS) de niños tratados por trastorno por déficit de atención con hiperactividad (TDAH) durante un corto período de tiempo y comparar las puntuaciones con las normas de referencia de la población general.

Métodos: Estudio prospectivo en niños de 6 a 12 años con TDAH. Los padres y sus hijos completaron la versión española del CHIP-CE (Child Health and Illness Profile-Child Edition 'Perfil de salud infantil') al iniciar el tratamiento y a las 8 semanas. Las puntuaciones del CHIP-CE de ambas visitas se compararon mediante el test de la $t$ de Student para datos apareados, el tamaño del efecto (TE), los coeficientes de correlación intraclase (CCI) y los diagramas de dispersión. Las evaluaciones de padres e hijos se compararon con las puntuaciones del CHIP-CE de la muestra de referencia española.

Resultados: En el análisis se incluyó a 31 niños y a sus padres. El mayor cambio entre la visita inicial y la visita de seguimiento se produjo en la dimensión de riesgos, tanto en los niños como en los padres ( $T E=0,24$ y 0,49 , respectivamente). $\mathrm{El} \mathrm{CCl}$ presentó un intervalo de entre 0,44 (satisfacción) y 0,01 (riesgos). Las puntuaciones de los niños fueron similares a los valores de referencia poblacional. Todas las dimensiones de la versión de padres del CHIP-CE presentaron puntuaciones medias estandarizadas inferiores a los valores de
\end{abstract}

\footnotetext{
*Autor para correspondencia.

Correos electrónicos: Irajmil@aatrm.catsalut.net, Irajmil@imim.es (L. Rajmil).
} 


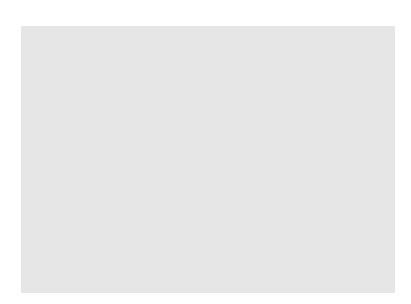

referencia en la visita inicial y fueron próximas a los valores de referencia tras el tratamiento.

Conclusiones: El presente estudio mostró poca concordancia entre padres e hijos y sugiere que se deberían recoger ambas perspectivas en futuros estudios del impacto y del tratamiento del TDAH.

(C) 2008 Asociación Española de Pediatría. Publicado por Elsevier España, S.L. Todos los derechos reservados.

Parent-child agreement in the health related quality of life (HRQOL) of children with attention-deficit/hyperactivity disorder (ADHD): A longitudinal study

\begin{abstract}
Objectives: To assess parent-child agreement on changes over a short-term period of time in the HRQOL of children treated for ADHD over a short period of time, and to compare child and parent ratings of children with ADHD with general population norms.

Methods: Prospective study in children 6-12 years old with ADHD. Children and parents completed the Spanish versions of the Child Health and Illness Profile-Child Edition (CHIPCE) before and after 8 weeks of treatment. CHIP-PE scores at both visits were compared using paired t tests and effect sizes (ES), intra-class correlation coefficients (ICC), and scatter plots. Child and parent ratings were compared with CHIP-CE scores for a general population sample.

Results: Thirty-one children and parents were included in the analysis. The highest change between the first and the follow-up visit was on the Risk Avoidance domain both children and parents (effect size [ES] $=0.24$ and 0.40 , respectively). The ICC ranged from 0.44 (Satisfaction) to 0.01 (Risk avoidance). Child self-ratings were close to general population values. All domains of the parent version presented standardized means below the reference values at the baseline visit and closer to the general population norm after treatment.

Conclusions: This study found poor parent-child agreement and suggests that both ratings should be collected in future studies on the impact of ADHD and treatment effectiveness. (C) 2008 Asociación Española de Pediatría. Published by Elsevier España, S.L. All rights reserved.
\end{abstract}

\section{Introducción}

El trastorno por déficit de atención con hiperactividad (TDAH) es una alteración caracterizada por falta de concentración, hiperactividad e impulsividad, y presenta un impacto negativo y perjudicial en el desarrollo del niño ${ }^{1}$. Según los estudios publicados, la prevalencia depende del período y del lugar donde se ha llevado a cabo el estudio así como del procedimiento diagnóstico empleado. Las estimaciones varían entre el 4 y el $12 \%$ en la población general de niños escolarizados en educación primaria, con resultados similares en estudios llevados a cabo en centros de atención primaria $^{2-4}$. Se han encontrado prevalencias más elevadas cuando la información se obtuvo de los padres mediante la aplicación de los criterios del Diagnostic and Statistical Manual of Mental Disorders (DSM-IV) ${ }^{1}$ que cuando los datos se obtuvieron de los propios niños mediante el Diagnostic Interview Schedule for Children ${ }^{5}$.

Los instrumentos multidimensionales que miden la calidad de vida relacionada con la salud (CVRS) permiten evaluar la salud de los niños a partir de dimensiones físicas, psicológicas y sociales $^{6-8}$. Los estudios de la CVRS en una población afectada de TDAH han mostrado que los padres perciben un impacto significativo en el estado de salud del niño, así como en las actividades y el funcionamiento familiares, y que el TDAH se asocia a problemas emocionales en los padres. Los padres de niños con TDAH declaran más problemas de comportamiento y psicosociales en sus hijos que aquellos padres de hijos con asma?.

Pocos estudios han analizado la CVRS de niños con TDAH mediante instrumentos autoadministrados ${ }^{10-12}$. Uno de los principios de la evaluación de la CVRS es la autopercepción que tienen los individuos de su propia salud y bienestar, por lo que hay razones para cuestionar el uso de los padres como informadores indirectos cuando es posible recoger información de los propios niños. Hay pruebas de que la percepción que tienen los padres de la enfermedad de sus hijos tiene un mayor impacto de lo que señalan los propios niños y que la concordancia entre padres e hijos, en general, es menor en aquellas dimensiones de salud menos «observables», como la función emocional o social ${ }^{6}$. Un estudio en niños con TDAH mostró una correlación moderada entre las puntuaciones de padres e hijos en 5 de las 8 dimensiones del Child Health Questionnaire $(\mathrm{CHQ})^{11}$. Este estudio también mostró que mientras los padres puntuaron la salud de sus hijos sustancialmente peor respecto a los valores de referencia 
de la población general, las puntuaciones de los propios niños eran muy próximas a las normas poblacionales. Como han sugerido otros autores, hay razones para obtener información, siempre que sea posible, tanto de padres como de sus hijos 6 . Por otra parte, pocos estudios han analizado la CVRS de forma longitudinal en pacientes que reciben tratamiento para el TDAH y la mayoría de los estudios se centran en los niños mayores y adolescentes. Los objetivos del presente estudio fueron evaluar la concordancia entre padres e hijos sobre los cambios producidos en la CVRS de niños tratados por TDAH durante un corto período de tiempo y comparar las puntuaciones con las normas de referencia de la población general, para esto se empleó la versión española del CHIP-CE (Child Health and Illness Profile-Child Edition 'Perfil de salud infantil').

\section{Muestra del estudio}

Los pacientes se seleccionaron en el servicio de psiquiatría infantil de un hospital universitario de Barcelona y en un centro de salud mental infantojuvenil para pacientes ambulatorios adyacente al hospital mencionado. Entre septiembre y noviembre de 2003 se incluyó de forma consecutiva a los pacientes que cumplían los siguientes criterios de inclusión: 1) pacientes diagnosticados de TDAH por un psiquiatra según criterios del $\mathrm{DSM}^{-I^{1}}{ }^{1} ; 2$ ) pacientes que nunca se habían tratado con psicoestimulantes (metilfenidato $u$ otro) $o$ pacientes que no lo habían recibido durante el período de vacaciones estivales (desde finales de junio hasta mediados de septiembre), y 3) pacientes entre 6 y 12 años de edad. Los criterios de exclusión fueron los siguientes: 1) alteraciones motoras o perceptivas; 2 ) retraso mental definido (coeficiente intelectual menor de 70); 3) autismo; (4) psicosis, y 5) cambio de centro de salud durante el transcurso del estudio. El Comité de Ética e Investigación del hospital de Sant Joan de Déu de Barcelona aprobó el protocolo del estudio y se obtuvo el consentimiento informado de todos los padres o tutores de los niños que participaron en el estudio.

\section{Diseño del estudio}

Se trata de un estudio prospectivo, pretratamiento y postratamiento, observacional, de un único grupo, realizado en niños en proceso de inicio o de reanudación de su tratamiento con metilfenidato para el TDAH. En la visita de selección, inmediatamente después de las vacaciones escolares (visita 0), los psiquiatras (Alda e Izaguirre) confirmaron el diagnóstico de TDAH y establecieron el régimen de tratamiento inicial más adecuado (nuevos casos) o la renovación del tratamiento con metilfenidato (tratamiento interrumpido). En esta visita, los psiquiatras solicitaron la participación de padres e hijos en el estudio y recogieron las variables sociodemográficas y clínicas. Se concretó una visita posterior (visita 1) con una media de 16 días después de la selección para la administración de los cuestionarios del estudio. Se asumió que el metilfenidato aún no había sido efectivo en la visita 1 . Se concretó una visita de control (visita 2) 8 semanas después de la visita 1 para evaluar el cambio en la CVRS durante este tiempo. Los niños incluidos en el presente estudio recibieron tratamien- to con metilfenidato, que se ha recomendado para el TDAH en la infancia y en la juventud ${ }^{13,14}$ tanto en España ${ }^{16-18}$ como en el resto de Europa ${ }^{15}$. El tratamiento con metilfenidato ha demostrado su eficacia en la reducción de síntomas en niños en edad escolar ${ }^{19}$.

\section{Medidas}

Fue la versión española del CHIP-CE; el CHIP-CE/Child Report Form (versión infantil del CHIP-CE) (CHIP-CE/CRF) ${ }^{20}$ mide la salud autopercibida de niños con edades entre 6 y 12 años e incluye 44 ítems en 5 dimensiones (satisfacción, bienestar, resistencia, riesgos y funciones). Las preguntas contienen 5 opciones de respuesta en una escala Likert con círculos graduados (p. ej., nunca, casi nunca, algunas veces, casi siempre o siempre). De los 44 ítems, 28 se enmarcan en un período recordatorio de 4 semanas. El CHIP-CE/Parent Report Form (versión para padres del CHIP-CE) (CHIP-CE/ $\mathrm{PRF})^{21}$ contiene 75 ítems. Además de las 5 dimensiones de la versión infantil, es posible generar 12 subdimensiones: satisfacción con la salud y autoestima (satisfacción), bienestar físico, bienestar emocional y limitación de actividad (bienestar), participación familiar, actividad física y resolución de problemas (resistencia), riesgo individual y amenazas a logros (riesgos) y rendimiento académico y relaciones con pares (funciones). Las respuestas se recogen en la escala Likert de 5 categorías. Las puntuaciones más elevadas indican un mejor estado de salud (mayor satisfacción, mayor bienestar, mayor resistencia, menor riesgo y mejor función).

El CHIP-CE se ha adaptado en España siguiendo un procedimiento de traducción directa e inversa ${ }^{22}$ y ha demostrado aceptable fiabilidad y validez ${ }^{23,24}$. Con el fin de facilitar la interpretación, las puntuaciones de las dimensiones y de las subdimensiones se estandarizaron a una media de 50 con una desviación estándar (DE) de 10 y se utilizaron para esto las puntuaciones de referencia de la muestra de la población española. Los valores de referencia se obtuvieron en un estudio llevado a cabo en escuelas de primaria de Barcelona durante el curso académico de 2002 a 2003.

Los cuestionarios del estudio se administraron a grupos de entre 5 y 10 niños, con la ayuda de un entrevistador en los niños de 6 y de 7 años y autoadministrados en los niños de más edad. Los padres completaron su versión del cuestionario en una sala contigua. En la visita 2 se administraron los mismos cuestionarios siguiendo el procedimiento de la visita anterior.

\section{Características clínicas y sociodemográficas}

Otras variables recogidas fueron género, edad, centro (hospital o consulta externa), relación del adulto con el niño (padre o madre), tipo de TDAH (predominio de hiperactividad, predominio de déficit de atención o combinación de ambas), comorbilidad psiquiátrica (sí/no y tipo), TDAH tratado con metilfenidato antes de las vacaciones escolares (sí/no), otros tratamientos (para trastornos de conducta y otras enfermedades) y tiempo transcurrido desde el diagnóstico del TDAH (en años, categorizado como menos de un año, un año y más de un año). 


\section{Análisis estadístico}

Se llevo a cabo una comparación de las puntuaciones de padres e hijos en la visita inicial mediante el test de la t de Student y se evaluaron los cambios en las puntuaciones entre las visitas del estudio mediante el test de la $t$ de Student para datos apareados. La magnitud del cambio del CHIP-CE/CRF y del CHIP-CE/PRF se analizó utilizando el tamaño del efecto (TE) de Cohen ${ }^{25,26}$. Los TE de 0,2 a 0,5; de 0,51 a 0,8 y superiores a 0,8 se consideraron mínimos, moderados e importantes, respectivamente. El diagrama de dispersión de Bland y Altman ${ }^{27}$ se utilizó para determinar la representación visual del grado de acuerdo entre padre e hijo. Se calcularon las puntuaciones medias estandarizadas y sus intervalos de confianza (IC) del 95\% con el fin de comparar las puntuaciones de padres e hijos con sus valores de referencia de la población general para las dimensiones del CHIP-CE. Se comprobó la fiabilidad a partir de valores $\alpha$ de Cronbach para cada dimensión del CHIP-CE, tanto para la visita inicial como para la visita de seguimiento. Se consideraron satisfactorios los valores $\alpha$ de Cronbach mayores o iguales a $0,70^{28}$. Se estimaron los coeficientes de correlación intraclase $(\mathrm{CCl})$ para evaluar el acuerdo entre padre e hijo en la visita inicial, en la visita de seguimiento y los cambios producidos entre ambas visitas. El grado de acuerdo se consideró bajo (inferior a 0,2); regular (de 0,21 a 0,40 ); moderado (de 0,41 a 0,60 ); sustancial (de 0,61 a 0,8 ), y excelente (superior a 0,8$)^{29}$.

\section{Resultados}

De los 55 niños con TDAH seleccionados inicialmente para su inclusión en el estudio, 33 cumplieron con los criterios de selección y todos aceptaron participar. Los motivos de exclusión se debieron a cambios de los pacientes o a otras causas que motivaron un cambio de centro de salud antes de la primera visita (15 casos), a la negativa de los padres a recibir psicoestimulantes (6 casos), a retraso mental (un caso) y a psicosis (un caso). Dos de los niños no acudieron a la visita inicial. En la visita inicial se incluyó a 31 niños para su análisis. Cuatro de los niños no completaron las visitas de seguimiento y se incluyó a un total de 27 casos en los análisis de ambas visitas.

La tabla 1 muestra las características clínicas y sociodemográficas de la muestra. La media de edad fue de 9,4 años (DE de 1,8$)$ y 28 eran niños $(90 \%)$. El tipo de TDAH combinado fue el más frecuente $(61 \%)$ y el tiempo desde el diagnóstico fue de 2,03 años (rango de 0 a 6 años). Diecinueve casos (61\%) habían recibido psicoestimulantes antes aunque estuvieron libres de tratamiento durante una media de 72 días (rango de 40 a 92 días) durante el período de vacaciones escolares. Las comorbilidades psicosociales fueron trastorno con conducta oposicionista y desafiante $(n=16)$, trastorno de conducta $(n=12)$, trastorno depresivo $(n=2)$, retraso específico de aprendizaje $(n=1)$ y terrores nocturnos $(n=1)$. De éstos, 8 casos con trastornos de conducta recibían tratamiento (neurolépticos: $n=4$; comportamental: $n=2$, o asesoramiento paterno: $n=2$ ).

La tabla 2 muestra las puntuaciones (medias y DE) no estandarizadas del CHIP-CE/CRF y del CHIP-CE/PRF en la visita inicial y en la visita de seguimiento, así como las
Tabla 1 Características de la muestra de niños con trastorno por déficit de atención con hiperactividad en la visita de selección $(n=31)$

\begin{tabular}{lc} 
Pacientes $(n=31)$ \\
\hline$n$ & $\%$ \\
8 & 25,8 \\
23 & 74,2
\end{tabular}

Sexo

Niños $\quad 28 \quad 90,3$

Niñas $\quad 3 \quad 9,7$

Lugar de selección

$\begin{array}{lll}\text { Hospital } & 10 & 32,2 \\ \text { Centro de salud mental } & 21 & 67,8\end{array}$

infantojuvenil

Persona que responde

$\begin{array}{lll}\text { Madre } & 23 & 76,7 \\ \text { Padre } & 4 & 13,3 \\ \text { Ambos } & 3 & 10,0\end{array}$

$\begin{array}{lll}\text { Tipo de TDAH } & & \\ \text { Hiperactividad } & 5 & 16,1 \\ \text { Déficit de atención } & 7 & 22,6 \\ \text { Combinado } & 19 & 61,3\end{array}$

Tiempo desde el diagnóstico (años) hasta la visita 1

$\begin{array}{lll}\text { Inferior a un año } & 6 & 19,4 \\ \text { Un año } & 9 & 29,0 \\ \text { Superior a un año } & 16 & 51,6\end{array}$

Tratamiento previo con metilfenidato

$\begin{array}{lll}\text { Sí } & 19 & 61,3 \\ \text { No } & 12 & 38,7\end{array}$

Comorbilidad psiquiátrica

$\begin{array}{lll}\text { Sí } & 16 & 51,6 \\ \text { No } & 15 & 48,4\end{array}$

TDAH: trastorno por déficit de atención con hiperactividad.

diferencias de puntuación entre las visitas y los valores $\alpha$ de Cronbach de cada una de las 5 dimensiones. En la autoevaluación de los niños no hubo cambios estadísticamente significativos en la puntuación de ninguna de las dimensiones del CHIP-CE entre las visitas. Los TE fueron menores o iguales a 0,2 para las puntuaciones de los niños. En la CHIPCE/PRF se apreció una mejora estadísticamente significativa $(p<0,01)$ en la dimensión de riesgos. El TE más elevado tanto en niños como en padres se observó en la dimensión de riesgos $(0,24$ y 0,49 , respectivamente). Hubo cambios estadísticamente significativos con mejores puntuaciones a las 8 semanas después del tratamiento en las siguientes 
Tabla 2 Puntuaciones brutas (media y desviación estándar) de las dimensiones del Perfil de salud infantil,, tamaños del efecto y valores $\alpha$ de Cronbach para los niños y los padres en la visita inicial y en la visita de seguimiento

\begin{tabular}{|c|c|c|c|c|c|c|c|}
\hline & \multicolumn{3}{|c|}{ Visita inicial } & \multicolumn{3}{|c|}{ Visita de seguimiento } & \multirow{2}{*}{$\begin{array}{l}\text { Tamaño del } \\
\text { efecto }\end{array}$} \\
\hline & Media & $\mathrm{DE}$ & $\begin{array}{l}\text { Valores } \alpha \text { de } \\
\text { Cronbach }\end{array}$ & Media & $\mathrm{DE}$ & $\begin{array}{l}\text { Valores } \alpha \text { de } \\
\text { Cronbach }\end{array}$ & \\
\hline \multicolumn{8}{|c|}{ Versión infantil (CHIP-CE/CRF) } \\
\hline Satisfacción & 4,29 & $(0,54)$ & 0,78 & 4,27 & $(0,68)$ & 0,88 & 0,02 \\
\hline Bienestar & 4,31 & $(0,47)$ & 0,72 & 4,31 & $(0,43)$ & 0,67 & 0,04 \\
\hline Resistencia & 3,79 & $(0,76)$ & 0,74 & 3,91 & $(0,87)$ & 0,84 & 0,07 \\
\hline Riesgos & 3,69 & $(0,85)$ & 0,82 & 3,82 & $(0,71)$ & 0,70 & 0,24 \\
\hline Funciones & 4,31 & $(0,59)$ & 0,75 & 4,26 & $(0,59)$ & 0,65 & $-0,01$ \\
\hline \multicolumn{8}{|c|}{ Versión de padres (CHIP-CE/PRF) } \\
\hline Satisfacción & 3,66 & $(0,77)$ & 0,84 & 3,73 & $(0,68)$ & 0,88 & 0,21 \\
\hline Bienestar & 4,13 & $(0,37)$ & 0,85 & 4,22 & $(0,41)$ & 0,87 & 0,20 \\
\hline Resistencia & 3,75 & $(0,37)$ & 0,67 & 3,83 & $(0,41)$ & 0,75 & 0,27 \\
\hline Riesgos & 3,75 & $(0,44)$ & 0,81 & 3,92 & $(0,38)$ & 0,73 & $0,49^{*}$ \\
\hline Funciones & 3,42 & $(0,73$ & 0,83 & 3,45 & $(0,65)$ & 0,81 & 0,13 \\
\hline
\end{tabular}

CHIP-CE/CRF: Child Health and Illness Profile-Child Edition/Child Report Form 'versión infantil del Perfil de salud infantil'; CHIP-CE/ PRF: Child Health and Illness Profile-Child Edition/Parent Report Form ‘versión para padres del Perfil de salud infantil’; DE: desviación estándar.

${ }^{*} \mathrm{p}<0,01$, test de la t de Student para datos apareados.

Tabla 3 Concordancia entre padres e hijos en las puntuaciones del Perfil de salud infantil en la visita inicial, en la visita de seguimiento y diferencias entre ambas visitas

\begin{tabular}{llll}
\hline CCl (visita inicial) & $\mathrm{CCl}$ (visita de seguimiento) & $\begin{array}{l}\mathrm{CCl} \text { (diferencias entre la visita } \\
\text { inicial y la visita de seguimiento) }\end{array}$ \\
\hline Satisfacción & 0,44 & 0,39 & 0,23 \\
Bienestar & 0,15 & 0,37 & 0,28 \\
Resistencia & 0,05 & 0,37 & 0,32 \\
Riesgos & 0,11 & 0,01 & 0,07 \\
Funciones & 0,31 & 0,34 & 0,23 \\
\hline
\end{tabular}

CCl: coeficiente de correlación intraclase.

subdimensiones: bienestar emocional (DE de 0,47; $p<0,01$ ), resolución de problemas ( $D E$ de 0,$34 ; p<0,01)$, riesgo individual ( $D E$ de 0,$54 ; \mathrm{p}<0,01)$ y relaciones con pares (DE de 0,$25 ; p<0,01$ ) (datos no mostrados). Los valores $\alpha$ de Cronbach fueron satisfactorios en todas las dimensiones, tanto en la versión de padres como en la versión infantil.

La concordancia entre padres e hijos fue baja o regular en casi todas las dimensiones y permaneció relativamente estable en ambas visitas (tabla 3; $\mathrm{CCl}$ de 0,01 a 0,44). La concordancia entre padres e hijos fue ligeramente superior para satisfacción y bienestar en comparación con el resto de las dimensiones.

La figura 1 ( $A$ y B) muestra los diagramas de dispersión para las dimensiones de riesgos y funciones en la primera visita y en la visita de seguimiento. Los padres puntuaron peor que sus hijos en las 2 visitas y el grado de desacuerdo tendía a incrementarse en las dimensiones con peores puntuaciones.

La figura 2 (A y B) muestra las medias estandarizadas para el CHIP-CE/CRF y el CHIP-CE/PRF comparadas con las puntuaciones de referencia de la población general y su IC del $95 \%$. En general, las puntuaciones de los niños estaban muy próximas a los valores de la población general, excepto en riesgos (puntuación media de 45,14; IC del 95\%: de 40,77 a 49,48). La relación entre las puntuaciones de los niños y los valores de referencia de la población general permaneció prácticamente igual después del tratamiento aunque ya no había diferencias estadísticamente significativas en la dimensión riesgos. La figura $2 \mathrm{~B}$ muestra las puntuaciones de los padres. Todas las dimensiones de la versión de padres presentaron medias estandarizadas inferiores a los valores de referencia en la visita inicial (IC del 95\% superior a menos 

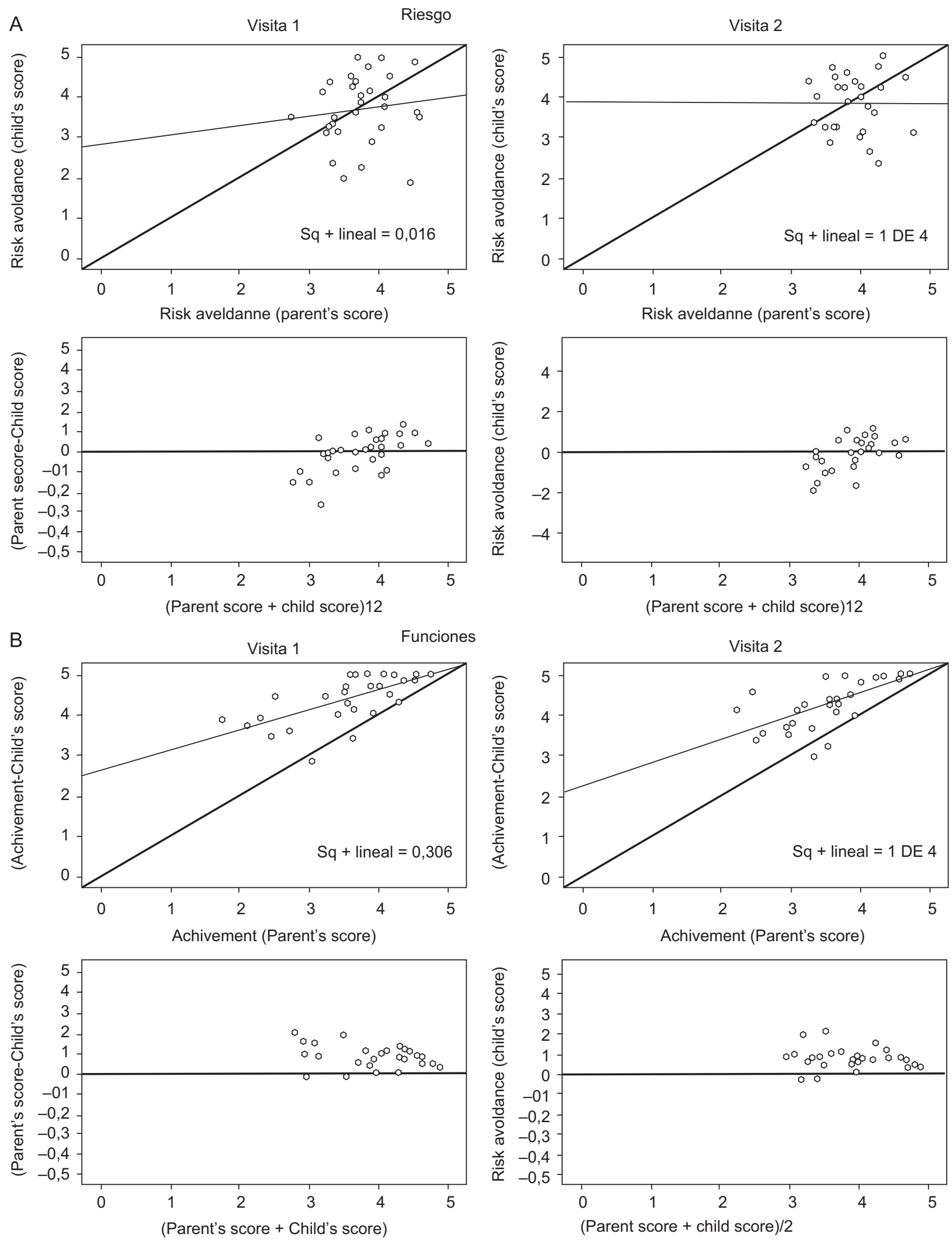

Figura 1 (A y B) Diagramas de dispersión de las puntuaciones de padres e hijos de las dimensiones de riesgos y funciones del Perfil de salud infantil. 
Media población general $=50$

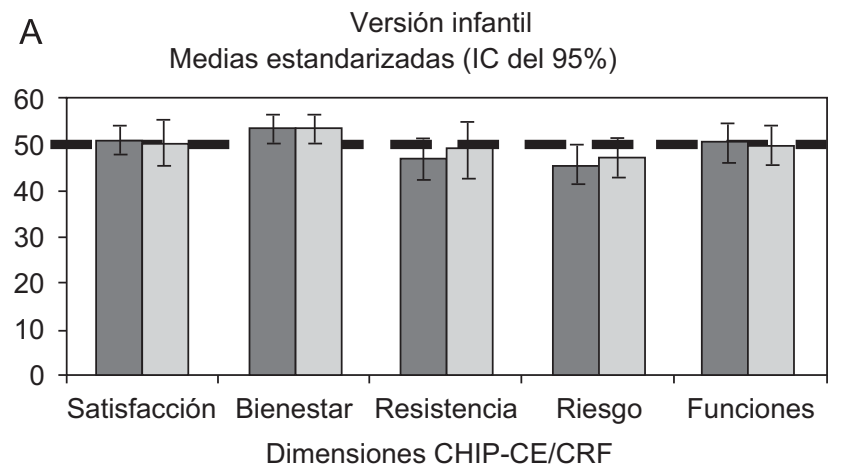

$\square$ Visita $1 \quad \square$ Visita 2
B Versión infantil

Medias estandarizadas (IC del 95\%)

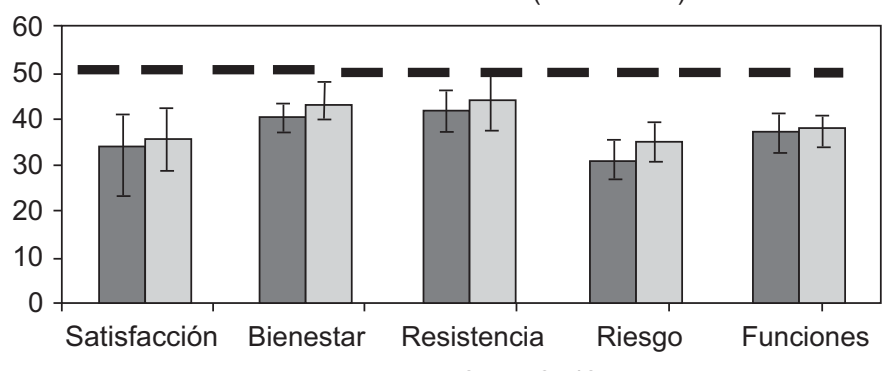

Dimensiones CHIP-CE/CRF

Visita $1 \quad \square$ Visita $2 \quad(\mathrm{n}=27)$

Figura 2 (A y B) Puntuaciones medias estandarizadas de la versión infantil y de padres del Perfil de salud infantil en la visita inicial (visita 1), después de 8 semanas de tratamiento (visita 2) y tamaño del efecto. Media poblacional de 50, 1 desviación estándar de 10.

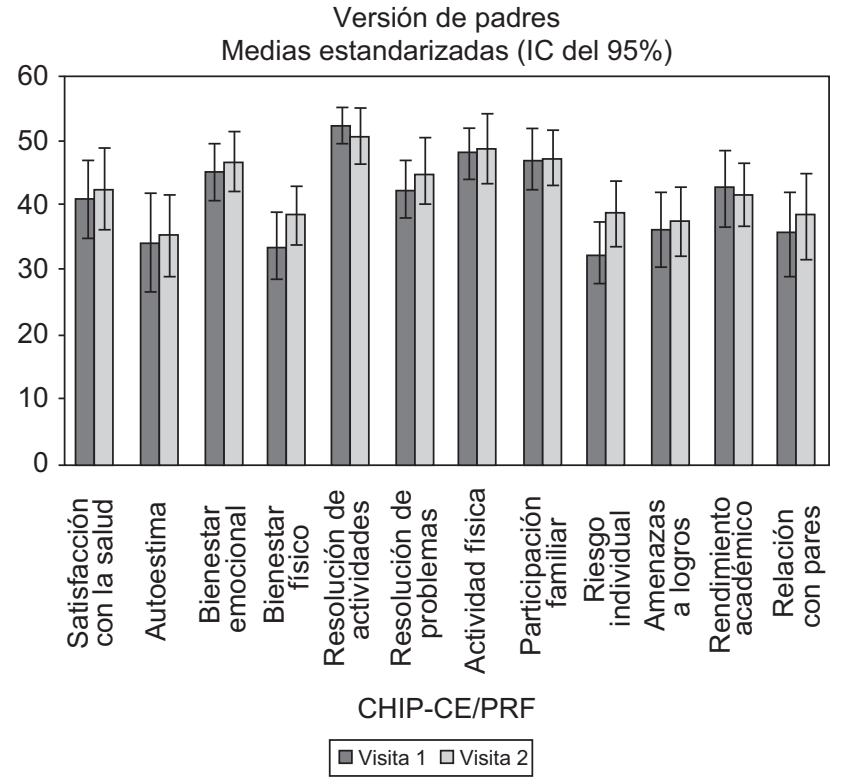

Figura 3 Puntuaciones medias estandarizadas de las subdimensiones de la versión de padres del Perfil de salud infantil en la visita inicial (visita 1), después de 8 semanas de tratamiento (visita 2) y tamaño del efecto. Media poblacional de 50, 1 desviación estándar de 10.

de 50 en todos los casos). Las puntuaciones de los padres en la segunda visita se aproximaron a los valores de la población general aunque seguían siendo inferiores a la media poblacional de 50 .

La figura 3 muestra las puntuaciones medias estandarizadas para el CHIP-CE/PRF en las subdimensiones comparadas con las medias de la población general. Las puntuaciones de los padres fueron inferiores a las medias de la población general a excepción de las siguientes subdivisiones: limitación de actividad, actividad física y participación familiar. En la visita de seguimiento, las puntuaciones medias se aproximaron a los valores de la población general en todas las subdimensiones y las diferencias estadísticamente significativas desaparecieron.

\section{Discusión}

El presente estudio es uno de los pocos dirigidos a medir la concordancia entre padres e hijos desde una perspectiva longitudinal en un corto período de tiempo en la CVRS de los niños con TDAH. La disponibilidad de valores de referencia poblacionales para el instrumento empleado en el estudio (CHIP-CE) permitió comparar las puntuaciones de padres e hijos con la población general antes y después del tratamiento. Se observó un acuerdo bajo entre padre e hijo en todas las dimensiones de salud tanto transversal como longitudinalmente. Los padres puntuaron peor la CVRS de sus hijos que los propios niños. Las puntuaciones de los padres sobre la CVRS de sus hijos mejoraron en la segunda visita. Por otro lado, las puntuaciones de los niños sobre su CVRS fueron próximas a los valores de referencia en la visita inicial, lo que dejó un menor margen de mejora en el seguimiento.

Los resultados del presente estudio confirman los hallazgos de un estudio reciente que examinó el acuerdo entre padre e hijo en la CVRS en niños con TDAH mediante el uso del $\mathrm{CHQ}^{11}$. Los autores de este estudio encontraron que los niños puntuaban su CVRS de forma muy similar a sus pares y que los padres puntuaban peor la CVRS de sus hijos en varias dimensiones del $\mathrm{CHQ}$; especialmente en el comportamiento general, la autoestima, la salud mental, la cohesión de la familia y las actividades. Sin embargo, ese estudió no analizó el cambio en la CVRS en el tiempo y se centró en niños de más edad y adolescentes. Una de las fortalezas del presente estudio es la obtención de valoraciones longitudinales de la CVRS procedentes de niños con una intervención común para el TDAH y la inclusión de niños a partir de los 6 años de edad.

Las diferencias encontradas en las puntuaciones de padres e hijos, como las observadas en el presente estudio, son relativamente frecuentes en la investigación de la CVRS en la población pediátrica ${ }^{30}$. Una posible explicación de 
estas diferencias es que los niños, en especial los niños más pequeños, no son conscientes de los síntomas y de las características que definen este trastorno, como la falta de concentración, la impulsividad, la hiperactividad, la desorganización y las dificultades en la consecución de las tareas. Es posible que los padres sean más conscientes que sus propios hijos de los cambios observables en el comportamiento de éstos o en los comportamientos que amenazan su competencia social, aunque es discutible si son más competentes a la hora de juzgar los sentimientos de su hijo que el propio niño. Los resultados del presente estudio se asemejan a los de estudios previos en los que los padres de hijos con trastornos crónicos puntuaron peor la CVRS que los propios niños, mientras que se ha encontrado la situación opuesta en poblaciones relativamente sanas ${ }^{31}$. Al mismo tiempo, los coeficientes de fiabilidad de las puntuaciones de los niños fueron satisfactorios, lo que indica la consistencia de sus respuestas. La valoración de la CVRS se basa en la medición de las impresiones subjetivas relacionadas con la salud de manera que las evaluaciones de los padres no pueden considerarse como único estándar dado que se evidencia que tienden a percibir la enfermedad con un mayor impacto que el que percibe el propio niño ${ }^{30}$.

Las dimensiones afectadas, en especial la de riesgos, es la esperada para esta población. La dimensión riesgos comprende los comportamientos que amenazan la competencia social de los niños, el desarrollo escolar y la interacción con sus pares. Cabe destacar que la subdimensión riesgo individual se vio especialmente afectada (según las puntuaciones de los padres) así como la autoestima, el bienestar emocional y las relaciones con pares. Este hecho es coherente con los resultados de otros estudios que han examinado, por ejemplo, las relaciones entre los síntomas del TDAH y la autoestima ${ }^{32}$ y la calidad de las relaciones de niños con diagnóstico de TDAH con sus pares ${ }^{33}$ y que también han confirmado los resultados de otros estudios que han investigado el impacto del TDAH en la $\mathrm{CVRS}^{8}$.

El presente estudio presentó una serie de limitaciones. En primer lugar, es probable que el tamaño relativamente pequeño de la muestra redujera la capacidad para detectar diferencias estadísticamente significativas entre los grupos. Sin embargo, el tamaño de la muestra fue suficiente para detectar diferencias de efecto (en el TE) de aproximadamente 0,8 , con un error $\beta$ de 0,2 y un error $\alpha$ de 0,05 . En segundo lugar, se utilizó una medida general de la CVRS en lugar de una medida específica para TDAH y, por tanto, es posible que se hayan recogido algunas áreas específicas de síntomas, de funcionamiento y de bienestar y que no hayan quedado reflejadas en los cambios producidos en el estado general de salud de esta población. Sin embargo, un trabajo previo ha demostrado la validez de estas medidas genéricas en numerosos trastornos de salud ${ }^{34}$. En tercer lugar, es posible que la muestra no sea representativa de la población con TDAH dado que los pacientes se identificaron en unidades de psiquiatría infantil, cuando en realidad hay muchos niños con TDAH que reciben tratamiento en centros de salud mental infantojuvenil y también en atención primaria. En cuarto lugar, tanto los padres como los hijos sabían que recibían el tratamiento. Este hecho hace difícil reconocer si los efectos (o la falta de efectos) reflejaron los cambios en la capacidad de los hijos o la confianza, tanto de los padres como de los hijos, en la efectividad del metilfenidato. Se asumió que no habría efecto entre el inicio de tratamiento y la primera administración del cuestionario. Si se hubiera producido este efecto, se podría haber reducido la posibilidad de mejora entre la primera visita y la visita siguiente. La literatura médica indica que la medicación comienza a tener efecto en una media de 4 semanas después del inicio del tratamiento. Finalmente, un período de seguimiento más largo podría haber dado la oportunidad de que los padres dieran mejores puntuaciones.

Como conclusión, en este estudio se encontró una baja concordancia en la percepción del estado de salud entre los padres y los niños con TDAH, tanto transversal como longitudinalmente. Los resultados indican que se deberían recoger ambas perspectivas en futuros estudios sobre el impacto del TDAH y la efectividad del tratamiento.

\section{Financiación}

Parcialmente subvencionado por el Fondo de Investigación Sanitaria del Ministerio de Sanidad y Consumo español (contrato No01/0420) y el CIBER de Epidemiología y Salud Pública CIBERESP.

\section{Agradecimiento}

A MD Estrada, Programa de Doctorado de la Universidad Autónoma de Barcelona.

\section{Bibliografía}

1. American Psychiatric Association (APA). Diagnostic and statistical manual of mental disorders (DSM-IV). 4th ed. Washington, DC (US): American Psychiatric Publishing, Inc; 1994.

2. Brown RT, Freeman WS, Perrin JM, Stein MT, Amler RW, Feldman $\mathrm{HM}$, et al. Prevalence and assessment of attention-deficit/ hyperactivity disorder in primary care settings. Pediatrics. 2001;107:e43.

3. Costello EJ, Edelbrock C, Costello AJ, Dulcan MK, Burns BJ, Brent D. Psychopathology in pediatric primary care: The new hidden morbidity. Pediatrics. 1988;82:415-24.

4. American Academy of Pediatrics, Committee on Quality Improvement and Subcommittee on Attention-Deficit/Hyperactivity Disorder. Clinical practice guideline: Diagnosis and evaluation of the child with attention-deficit/hyperactivity disorder. Pediatrics. 2000;105:1158-70.

5. Costello AJ, Edelbrock CS, Kalas R, Kessler MK, Klaric SA. National Institute of Mental Health. Diagnostic interview schedule for children. Bethesda, MD (US): National Institute of Mental Health; 1982.

6. Eiser C, Morse R. Quality-of-life measures in chronic diseases of childhood. Health Technol Assess. 2001;5:1-157.

7. Solans M, Pane S, Estrada MD, Serra-Sutton V, Berra S, Herdman $M$, et al. Health-related quality of life measurement in children and adolescents: A systematic review of generic and diseasespecific instruments. Value Health. 2007;11:742-64.

8. Klassen AF, Miller A, Fine A. Health-related quality of life in children and adolescents who have a diagnosis of attentiondeficit/hyperactivity disorder. Pediatrics. 2004;114:e541-7.

9. Escobar R, Soutullo CA, Hervas A, Gastaminza X, Polavieja P, Gilaberte I. Worse quality of life for children with newly diagnosed attention-deficit/hyperactivity disorder, compared with asthmatic and healthy children. Pediatrics. 2005;116: e364-9. 
10. Topolski TD, Edwards TC, Patrick DL, Varley P, Way ME, Buesching DP. Quality of life of adolescent males with attention-deficit hyperactivity disorder. J Atten Disord. 2004;7: 163-73.

11. Klassen AF, Miller A, Fine S. Agreement between parent and child report of quality of life in children with attention-deficit/ hyperactivity disorder. Child Care Health Dev. 2006;32:397-406.

12. Varni JW, Burwinkle TM. The PedsQL as a patient-reported outcome in children and adolescents with attention-deficit/ hyperactivity disorder: A population-based study. Health Qual Life Outcomes. 2006;4:26.

13. Scottish Intercollegiate Guidelines Network (SIGN). Attention deficit and hyperkinetic disorders in children and young people. A national clinical guideline. Issue 52. Edinburgh (United Kingdom): SIGN; 2001. Disponible en: http://www.sign.ac.uk/ pdf/sign52.pdf.

14. National Institute of Clinical Excellence (NICE). Guidance on the use of methylphenidate (Ritalin, Equasym) for attention deficit/ hyperactivity disorder (ADHD) in childhood. Technology Appraisal Guidance No 13. London (United Kingdom): NICE; 2000. Disponible en: http://nice.org.uk.

15. Taylor E, Döpfner M, Sergeant J, Asherson P, Banaschewski T, Buitelaar J, et al. European clinical guidelines for hyperkinetic disorder - first upgrade. Eur Child Adolesc Psychiatry. 2004;13: 17-I30.

16. Halasz G, Vance AL. Attention deficit hyperactivity disorder in children: moving forward with divergent perspectives. Med J Aust. 2002;177:554-7.

17. Agencia Española de Medicamentos y Productos Sanitarios (AEMPS). Rubifen [ficha técnica]. Madrid: AEMPS. Ministerio de Sanidad y Consumo; 2003. Disponible en: http://www.agemed.es.

18. Agencia Española de Medicamentos y Productos Sanitarios (AEMPS). Concerta [ficha técnica]. Madrid: AEMPS. Ministerio de Sanidad y Consumo; 2003. Disponible en: http://www. agemed.es.

19. Schachter HM, Pham B, King J, Langford S, Moher D. How efficacious and safe is short-acting methylphenidate for the treatment of attention-deficit disorder in children and adolescents: A meta-analysis. CMAJ. 2001;165:1475-88.

20. Riley AW, Forrest CB, Rebok GW, Starfield B, Green B, Robertson JA, et al. The child report form of the CHIP-Child edition: Reliability and validity. Med Care. 2004;42:221-31.

21. Riley AW, Forrest CB, Starfield B, Rebok GW, Robertson JA, Green BF. The parent report form of the CHIP-Child edition: Reliability and validity. Med Care. 2004;42:210-20.
22. Rajmil L, Serra-Sutton V, Estrada MD, Fernández de Sanmamed MJ, Guillamón I, Riley A, et al. Adaptación de la versión española del perfil de salud infantil. An Pediatr (Barc). 2004; 60:522-9.

23. Estrada MD, Serra-Sutton V, Tebé C, Alonso J, Rajmil L. Fiabilidad y validez de la versión española del perfil de salud infantil, CHIP-CE [comunicación oral en la XXII Reunión científica de la Sociedad Española de Epidemiología]. Gac Sanit. 2004;18:29.

24. Serra-Sutton V, Estrada MD, Rajmil L, Tebé C, Puig X, Alonso J. Valores poblacionales de referencia de la versión española del perfil de salud infantil, CHIP-CE [póster en la XXII Reunión científica de la Sociedad Española de Epidemiología]. Gac Sanit. 2004;18:57.

25. Cohen J. Statistical power analysis for the behavioral sciences. 2nd ed. Hillsdale, NJ (US): Lawrence Earlbaum Associates, Inc; 1988.

26. Casado A, Prieto L, Alonso J. El tamaño del efecto de la diferencia entre dos medias: ¿estadísticamente significativo o clínicamente relevante? Med Clin (Barc). 1999;112:584-8.

27. Bland JM, Altman DG. Statistical methods for assessing agreement between two methods of clinical measurement. Lancet. 1986;1:307-10.

28. Lohr KN. Assessing health status and quality-of-life instruments: Attributes and review criteria. Qual Life Res. 2002;11:193-205.

29. Szklo M, Nieto FJ. Epidemiology: Beyond the basics. Gaithersburg, MD (US): Aspen Publishers Inc; 2000.

30. Eiser C, Morse R. Can parents rate their child's health-related quality of life? Results of a systematic review. Qual Life Res. 2001;10:347-57.

31. Robitail S, Siméoni MC, Ravens-Sieberer U, Bruil J, Auquier P, or the KIDSCREEN Group. Children proxies' quality-of-life agreement depended on the country using the European KIDSCREEN52 questionnaire. J Clin Epidemiol. 2007;60:469-78.

32. Edbom T, Lichtenstein P, Granlund M, Larsson JO. Long-term relationships between symptoms of attention deficit hyperactivity disorder and self-esteem in a prospective longitudinal study of twins. Acta Paediatr. 2006;95:650-7.

33. Hoza B, Mrug S, Gerdes AC, Hinshaw SP, Bukowski WM, Gold JA, et al. What aspects of peer relationships are impaired in children with attention-deficit/hyperactivity disorder?. J Consult Clin Psychol. 2005;73:411-23.

34. Starfield B, Forrest CB, Ryan SA, Riley AW, Ensminger ME, Green BF. Health status of well vs ill adolescents. Arch Pediatr Adolesc Med. 1996;150:1249-56. 